\title{
SEARCH FOR CORONAL EMISSION LINES IN COOLING FLOW CLUSTERS WITH THE KECK 10 METER TELESCOPE ${ }^{1}$
}

\author{
L. YAN AND J. G. COHEN \\ Department of Astronomy, MS 105-24, California Institute of Technology, Pasadena, CA 91125; \\ ly@astro.caltech.edu, jlc@astro.caltech.edu \\ Received 1995 March 15; accepted 1995 May 31
}

\begin{abstract}
Using the Low Resolution Imaging Spectrograph on the Keck $10 \mathrm{~m}$ telescope, we have searched for the coronal emission line $[\mathrm{Fe} \mathrm{x}] 6374 \AA$ produced by the gas at temperatures of $10^{5}-10^{6} \mathrm{~K}$ in the central several $10 \mathrm{kpc}$ regions of two massive cooling flow clusters: Abell 1795 and PKS 0745-191. We did not detect any significant $[\mathrm{Fe} \mathrm{x}] 6374 \AA$ emission in either cluster. The $1 \sigma$ surface brightness limits for the $[\mathrm{Fe} \mathrm{x}] 6374 \AA$ line in the central $75 h_{50}^{-1} \mathrm{kpc}$ region of Abell 1795 and in the central $30 h_{50}^{-1} \mathrm{kpc}$ region of PKS $0745-191$ are $10^{-18} \mathrm{ergs} \mathrm{s}^{-1} \mathrm{~cm}^{-2} \operatorname{arcsec}^{-2}$ and $7 \times 10^{-19} \mathrm{ergs} \mathrm{s}^{-1} \mathrm{~cm}^{-2} \operatorname{arcsec}^{-2}$, respectively. Our upper limits of the $[\mathrm{Fe} \mathrm{x}] 6374 \AA$ surface brightness in both clusters are about a factor of 10 better than the previous measurements. These results are consistent with the theoretically predicted [Fe $\mathrm{x}] 6374 \AA$ surface brightness by the popular inhomogeneous models (Sarazin \& Graney 1991), in which gas cools out of the flow at a range of radii, leaving the hot gas distributed as $\dot{M} \propto r$. The upper limits on mass cooling rate derived from our measurement are also consistent with the cooling rates derived from X-ray data for these two clusters. Our results are contradictory to the claimed significant detection in Abell 1795 by Anton et al. (1991) and the $3 \sigma$ marginal detection in PKS 0745-191 reported by Donahue \& Stocke (1994).

In addition, we also measured upper limits on the $[\mathrm{Fe} \mathrm{x}] / \mathrm{H} \alpha$ ratio in both clusters, which provide strong constraints on various theoretical models of nebulae heating for production of the luminous low-ionization emission lines by cool $\left(\sim 10^{4} \mathrm{~K}\right)$ nebular filaments. The $[\mathrm{Fe} \mathrm{x}] / \mathrm{H} \alpha(R<15 \mathrm{kpc})$ ratios in Abell 1795 and PKS $0745-191$ are less than $0.6 \%$ and $0.13 \%$, respectively.
\end{abstract}

Subject headings: cooling flows - galaxies: clusters: general

\section{INTRODUCTION}

X-ray observations have revealed that clusters of galaxies are frequently among the most luminous X-ray sources in the sky. X-ray photons are primarily from thermal bremsstrahlung emission in hot, diffuse plasma at temperatures of $10^{7}-10^{8} \mathrm{~K}$. The inferred cooling timescale from the X-ray surface brightness profile for the hot plasma within the central $50-300 \mathrm{kpc}$ region in most clusters is less than a Hubble time (Edge, Stewart, \& Fabian 1992). This indicates that in some clusters, the hot plasma may be dense enough to cool and settle in the central region of the cluster. The detection of the Fe XVII line at $826 \mathrm{eV}$ from X-ray spectroscopic observations (Mushotzky 1992; Canizares, Markert, \& Donahue 1988; Mushotzky \& Szymkowiak 1988) also indicates that the hot gas does indeed cool from $10^{7}-10^{8} \mathrm{~K}$ to $T \leq 5 \times 10^{6} \mathrm{~K}$, losing more than $90 \%$ of its thermal energy in the observed X-ray band. The estimated cooling rate inferred from X-ray observations is typically about $10-500 \mathrm{M}_{\odot} \mathrm{yr}^{-1}$ (Edge et al. 1992). If hot gas in cooling flow clusters is indeed cooling with a rate of $100 M_{\odot}$ $\mathrm{yr}^{-1}$, within a Hubble time there should be $10^{12} M_{\odot}$ of cold gas dropping out of cooling flows. However, we do not detect such a tremendous amount of cold gas in clusters of galaxies. Thus the key question is whether indeed hot gas in cooling flow clusters is cooling with such a high rate.

Mass cooling rates inferred from X-ray observations depend heavily on the theoretical models of the temperature distribu-

\footnotetext{
${ }^{1}$ Based on observations obtained at the W. M. Keck Observatory, which is operated jointly by the California Institute of Technology and the University of California.
}

tion of the hot gas. A model-independent method, first pointed out by Cowie (1981), is to use optical coronal emission lines to probe the gas at temperatures of $10^{5.5}$ to $10^{6.5} \mathrm{~K}$. Since the gas in this temperature range cools very rapidly, it tends to be thermally unstable. So if the cluster gas at $10^{7}-10^{8} \mathrm{~K}$ indeed cools, it also cools through temperatures of $10^{5.5}$ to $10^{6.5} \mathrm{~K}$, where optical coronal lines are produced. The gas at temperatures $\left(10^{5.5}-10^{6.5} \mathrm{~K}\right)$ could also be produced by ways other than direct cooling from the hot gas phase. For example, the mixture of the hot gas $\left(10^{7} \mathrm{~K}\right)$ and the cool gas $\left(10^{4} \mathrm{~K}\right)$ can create the intermediate-temperature gas by turbulent selfabsorbed mixing layers (Begelman \& Fabian 1990). However, the intermediate-temperature gas in the "mixing layers" model should still emit optical coronal lines. The luminosity in a given coronal line is $\sim(5 / 2) k T \dot{M} / \mu$ times the fraction of the total cooling emissivity in that transition, where $\mu$ is the atomic weight of coronal line-emitting ions. The total [Fe x] $6374 \AA$ line luminosity is therefore $\dot{M}$ times an almost modelindependent factor (Sarazin \& Graney 1991; Cowie 1981), i.e.,

$$
L_{[\mathrm{Fe} \mathrm{X}] 6374 \AA}=\left(2.1 \times 10^{+37}\right) \dot{M} \operatorname{ergs~s}{ }^{-1}\left(M_{\odot} \mathrm{yr}^{-1}\right)^{-1},
$$

The above equation includes the effect of nonequilibrium cooling (Voit, Donahue, \& Slavin 1994), where $\dot{M}$ is in units of $M_{\odot} \mathrm{yr}^{-1}$. Unfortunately the distribution of this luminosity on the sky, and thus the observable surface brightness, depends on the radius at which the gas cools and on the clumpiness of the cooling gas, as do the line profiles.

Detections of the $[\mathrm{Fe} \mathrm{x}] 6374 \AA$ emission line will provide an independent measurement of the large mass deposition rate in cooling flow clusters derived from X-ray observations. In this 
project, we searched for the [Fe x] $6374 \AA$ coronal emission line from gas at intermediate temperature of $10^{5.5}-10^{6.5} \mathrm{~K}$ in central galaxies in the cooling flow clusters Abell 1795 and PKS 0745-191. Both clusters have integrated mass cooling rates of $\sim 400 M_{\odot} \mathrm{yr}^{-1}$ within the cooling radii of $\sim 240 \mathrm{kpc}$ (Edge et al. 1992; Edge \& Stewart 1991). Edge et al. (1992) also calculated the curve of integrated mass cooling rate $\dot{M}$ versus radius $r$ from the cooling radius down to the central $40 \mathrm{kpc}$ in the cluster Abell 1795. This curve allows us later to make comparisons between $\dot{M}$ derived from coronal emission-line observations and X-ray observations. However, PKS 0745-191 does not have published X-ray observations down to the scale of a few $10 \mathrm{kpc}$. The properties of Abell 1795 and PKS 0745-191 are tabulated in Tables 1 and 2.

Several attempts have been made to search for optical coronal emission lines in nearby cooling flow clusters. The first detection of [Fe x] $6374 \AA$ was reported by Anton, Wagner, \& Appenzeller (1991). They claimed to have a $13 \sigma$ detection of $[\mathrm{Fe} \mathrm{X}] 6374 \AA$ in the central \pm 2 " around the nucleus of the $\mathrm{cD}$ galaxy in the cluster Abell 1795 . They measured the [Fe $\mathrm{x}]$ $6374 \AA$ line surface brightness in the nucleus to be $3.0 \times 10^{-17}$ ergs $\mathrm{s}^{-1} \mathrm{~cm}^{-2} \operatorname{arcsec}^{-2}$. The derived integrated mass cooling rate in the central region of \pm 2 " $(2.5 \mathrm{kpc}$ in radius $)$ around the nucleus is $190 \mathrm{M}_{\odot} \mathrm{yr}^{-1}$ with $H_{0}$ of $75 \mathrm{~km} \mathrm{~s}^{-1} \mathrm{Mpc}^{-1}$, which is much higher than the $30 M_{\odot} \mathrm{yr}^{-1}$ estimated from X-ray observations by Edge et al. (1992). However, recent observations by Donahue \& Stocke (1994) of the same cluster Abell 1795 did not detect any significant coronal line emission [Fe $\mathrm{x}$ ] $6374 \AA$, with the $3 \sigma$ surface brightness limit of $2.4 \times 10^{-17}$ ergs $\mathrm{s}^{-1} \mathrm{~cm}^{-2} \operatorname{arcsec}^{-2}$ in the central region of $3.7 \mathrm{kpc}$ radius around the nucleus. In the same paper, Donahue \& Stocke (1994) reported a marginal $3 \sigma$ detection of [Fe X] $6374 \AA$ in the massive cooling flow cluster PKS 0745-191, with the [Fe X] $6374 \AA$ surface brightness of $6.9 \times 10^{-18} \mathrm{ergs} \mathrm{s}^{-1} \mathrm{~cm}^{-2}$ $\operatorname{arcsec}^{-2}$ in the central \pm 2.5 region $\left(\sim 15 h_{50}^{-1} \mathrm{kpc}\right.$ in the diameter). The integrated mass cooling rate from X-ray observations is $\sim 400 M_{\odot} \mathrm{yr}^{-1}$ within the cooling radius of $240 \mathrm{kpc}$. Another recent sensitive search for [Fe X] $6374 \AA$ emission line was done by Shields \& Filippenko (1992). They obtained no detection in the Perseus cluster NGC 1275, with derived upper limits of $[\mathrm{Fe} \mathrm{X}] 6374 \AA$ surface brightness of $5 \times 10^{-18}$ ergs $\mathrm{s}^{-1} \mathrm{~cm}^{-2} \operatorname{arcsec}^{-2}$ in the $2^{\prime \prime} 3-40^{\prime \prime}$ off-nucleus region.

Furthermore, most cooling flow clusters have filaments of low-ionization emission lines within $\sim 10 \mathrm{kpc}$ of the central galaxies. The observed $\mathrm{H} \alpha$ line luminosity in most cases is one to three orders of magnitude higher than what is predicted from the rate of cooling flow, if each hydrogen atom emits one $\mathrm{H} \alpha$ photon as it cools and recombines. This discrepancy suggests that there has to be an energy source which reheats the gas to a temperature around $10^{4} \mathrm{~K}$ in order to produce the observed high $\mathrm{H} \alpha$ luminosities. There exists a host of models to explain the energy sources of emission-line nebulae and the physical connections between optical emission-line nebulae and cooling flows (Baum 1992). The measurements of [Fe X] $6374 \AA$ and the $[\mathrm{Fe} \mathrm{X}] / \mathrm{H} \alpha$ ratio will provide some differentiation between various theoretical models.

$H_{0}$ of $50 \mathrm{~km} \mathrm{~s}^{-1} \mathrm{Mpc}^{-1}$ is generally used as a matter of convention in calculations of mass cooling rates from $\mathrm{X}$-ray data. For the comparison purpose, we adopt the same value for $H_{0}$ in the rest of the paper.

\section{OBSERVATIONS}

Single long-slit spectra of the central galaxies in the massive cooling flow clusters Abell 1795 and PKS 0745-191 were taken with the Low Resolution Imaging Spectrograph at the Keck 10 m telescope (Mauna Kea, HI) (Oke et al. 1995). Complete observational information is given in Table 1 . We used a 1200 groove $\mathrm{mm}^{-1}$ grating with $1^{\prime \prime}$ or $1^{\prime \prime} 5$ wide and $3^{\prime}$ long spectrograph slits. The corresponding spectral resolution is $\sim 2 \AA$ and $\sim 2.8 \AA$. In addition, the spatial scale and the dispersion scale on the CCD are 0.21 pixel $^{-1}$ and $0.64 \AA$ pixel $^{-1}$, respectively. We observed the massive cooling flow clusters Abell 1795 and PKS 0745-191 along both the major and the minor axes of the $\mathrm{cD}$ galaxy nuclei. The atmospheric seeing during all the observations was excellent, about 0 ".8. In addition to the cooling flow clusters, we took spectra of two bright, nearby giant elliptical galaxies, NGC 4472 and NGC 7626, with the same instrumental setup. These two galaxies do not

TABLE 1

SUMMARY OF OBSERVING LOG

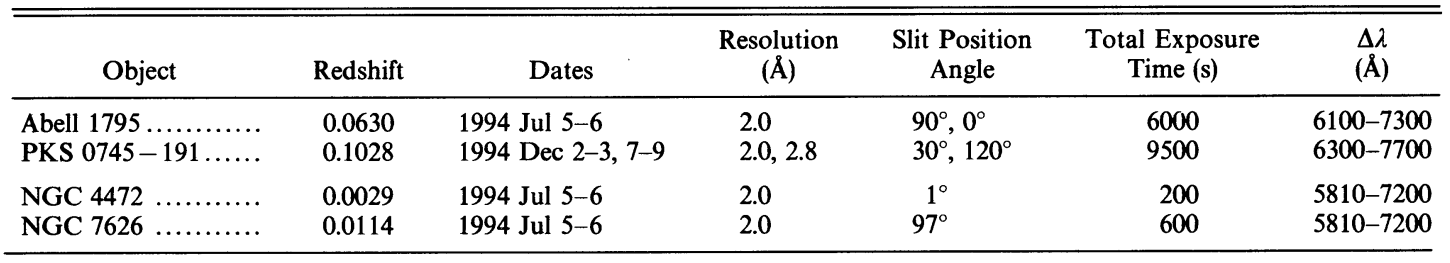

TABLE 2

Surface Brightness Limits and Mass CoOling Rates

\begin{tabular}{|c|c|c|c|c|c|c|}
\hline Object & $\underset{\left(e r g s \mathrm{~s}^{-1} \mathrm{~cm}^{-2} \operatorname{arcsec}^{-2}\right)}{S}$ & $\begin{array}{l}R_{\mathrm{FeX}} \\
(\mathrm{kpc})\end{array}$ & $\begin{array}{c}{[\mathrm{Fe} \mathrm{x}] / \mathrm{H} \alpha} \\
\text { (within } R_{\mathrm{Fe}} \text { ) }\end{array}$ & $\begin{array}{c}\dot{M}_{\mathrm{Fe} \mathrm{X}}\left(<R_{\mathrm{Fe} \mathrm{X}}\right) \\
\left(M_{\odot} \mathrm{yr}^{-1}\right)\end{array}$ & $\begin{array}{c}\dot{M}_{x}\left(<R_{c}\right) \\
\left(M_{\odot} \mathrm{yr}^{-1}\right)\end{array}$ & $\begin{array}{c}R_{\mathrm{c}} \\
(\mathrm{kpc})\end{array}$ \\
\hline Abell 1795 . & $10^{-18}$ & 15 & $0.6 \%$ & 170 & 410 & 270 \\
\hline PKS $0745-191 \ldots \ldots$ & $7 \times 10^{-19}$ & 15 & $0.13 \%$ & 115 & 430 & 230 \\
\hline
\end{tabular}

NoteS. $-S_{\mathrm{Fe} \mathrm{X}}$ is the surface brightness limit for [Fe X] $6374 \AA$ line. $R_{\mathrm{Fe}}$ is the radius of the central region over which $S_{\mathrm{Fe} X}$ is measured and the integrated mass cooling rate $\dot{M}_{\mathrm{Fe} \mathrm{X}}$ within this region is derived. $R_{c}$ is the X-ray derived cooling radius. Also for Abell 1795, we have a $1 \sigma$ limit map of the surface brightness profiles for [Fe x] $6374 \AA$ over the central region of $\sim 30$ kpc along both the major and minor axes. We adopt $H_{0}=50 \mathrm{~km} \mathrm{~s}^{-1} \mathrm{Mpc}^{-1}$. 
have strong emission lines. They serve as templates to subtract the stellar continua from the spectra of the central cD galaxy nuclei in the cooling flow clusters. Several faint spectrophotometric standard stars were also observed in order to obtain flux calibration. They are G191B2B, GD 248, Feige 34, $\mathrm{HZ}$ 4, and $\mathrm{HZ} 14$. The flux calibration for these stars is given in Oke (1990).

The CCD spectra were reduced using standard techniques in IRAF. For removal of the sky background, we choose regions far away from the nuclei and devoid of obvious nebular emission. Because of the long slit the night sky lines are not absolutely perpendicular to the dispersion axis. There is $\sim 1.3 \AA$ shift along the spectral direction across $65^{\prime \prime}$ along the spatial direction. Hence, the object, sky, and their corresponding neon arc comparison spectra are extracted with the same size aperture, and wavelength calibrated before we perform the sky subtraction. Figure $2 a$ shows the sky-subtracted spectrum of the central $2^{\prime \prime}$ nucleus of Abell 1795, Figure $2 b$ is the skysubtracted spectrum of the central $2^{\prime \prime}$ nucleus of PKS 0745-191 and Figure $2 c$ the sky-subtracted averaged spectrum of NGC 4472 and NGC 7626. Some differential features are caused by the imperfect sky subtraction, but these features do not affect the measurements described below.

\section{DATA ANALYSES}

\subsection{Abell 1795}

The centroid of the two-dimensional (2D) spectrum is traced along the dispersion direction. A total of 200 one-dimensional (1D) spectra are extracted out with the same aperture width of 1 pixel along the centroid of the $2 \mathrm{D}$ spectrum. The corresponding total spatial size is $21^{\prime \prime}$ in radius around the nucleus, e.g., $\sim 38 h_{50}^{-1} \mathrm{kpc}$ in radius, one-sixth of the cluster cooling radius. There are two reasons for using this approach. One is that it is not very clear how the coronal emission-line material is distributed, and whether it follows the optical emission-line nebulae. With these $2001 \mathrm{D}$ spectra we can obtain the spatial distribution of the coronal line emitting gas if there are any positive detections of [Fe x] $6374 \AA$. The second reason is that the distribution of emitting gas in the central galaxy of Abell 1795 is very lumpy and extended, and the radial velocity of the lumps varies significantly. Figure 1 (Plate 1) illustrates the 2D spectrum of Abell 1795. With the Fourier cross correlation technique (Tonry \& Davis 1979), we obtain the velocity map of the gas in the galaxy with the center as the zero point. Figure 3 shows that velocity map extending only to $\sim 7^{\prime \prime}$ radius from the galaxy center since for spectra beyond 7" there is not enough

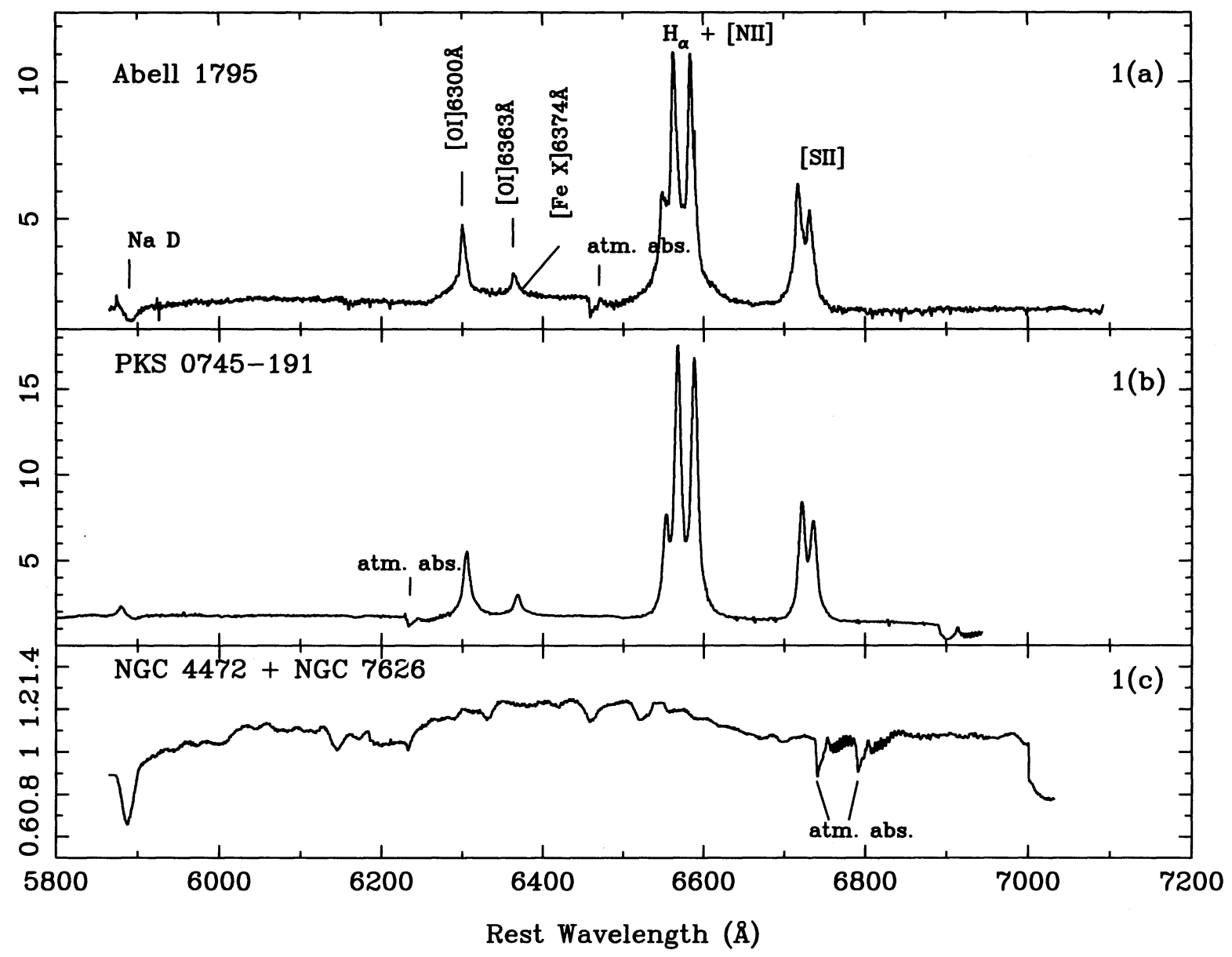

Fig. 2. - The top two panels show the spectra of the central cD galaxies in the massive cooling flow clusters Abell 1795 and PKS 0745-191. The coronal emission line $[\mathrm{Fe} \mathrm{X}] 6374 \AA$ is located on the red wing of the strong nebular emission features [ $\left.\mathrm{O}_{\mathrm{I}}\right] 6363 \AA$. The bottom panel is the averaged spectrum of NGC 4472 and NGC 7626 which are typical giant ellipticals without any emission lines. They are used as templates to subtract the features caused by underlying star light in the continua of the cooling flow galaxies. 




FIG. 1.-A two-dimensional long-slit spectrum of Abell 1795 including the $\mathrm{H} \alpha$ and [N II] emission lines along the minor axis. These high signal-to-noise data show that the nebular filaments in the central region of Abell 1795 have an extended spatial distribution and complex kinematic motions.

YAN \& COHEN (see 454, 46) 


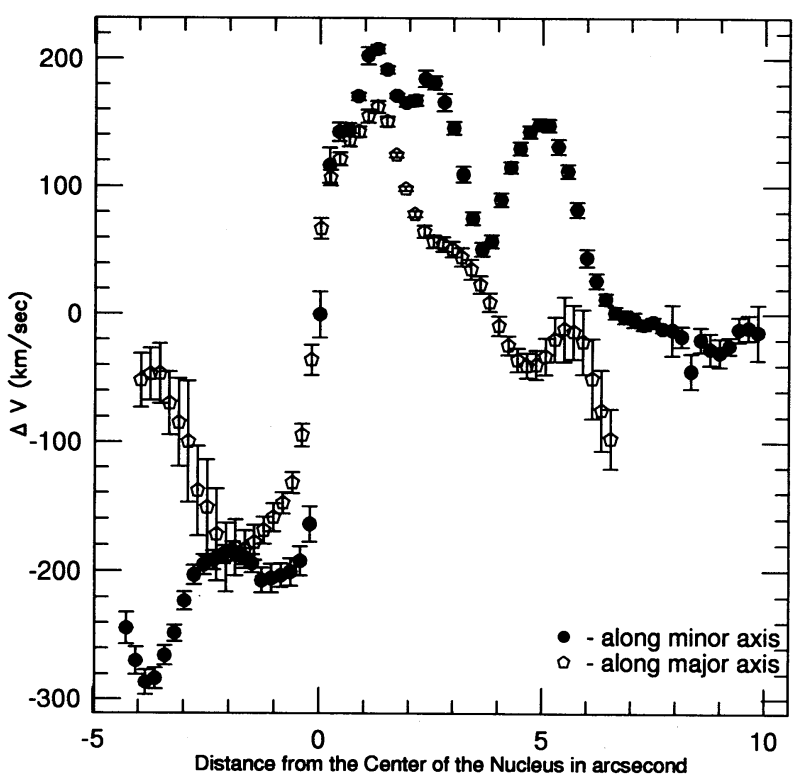

FIG. 3.-These are the rotation velocity curves for the emission-line gas in Abell 1795. The curve with solid dots is along the major axis, and the curve with open dots is along the minor axis. Each point represents the velocity at one position relative to the center of the nucleus. signal in the emission lines to compute the relative velocities. The range of the velocities is $500 \mathrm{~km} \mathrm{~s}^{-1}$. If the [Fe x] $6374 \AA$ emission-line gas follows the kinematics of the optical emission-line nebulae, a simple sum of all single 1D spectra would smear the weak [Fe $\mathrm{x}]$ emission line and make it harder to detect. Hence we group the 1D spectra according to their kinematics.

The elliptical galaxies NGC 4472 and NGC 7626 were observed to serve as the templates for subtracting the continuum from the spectrum of Abell 1795. Figure $2 c$ shows the averaged template spectrum. The features in the continua in Figure $2 a, 2 b$, and $2 c$ are caused by the underlying starlight in the galaxies. It is apparent that the underlying continua of the cD galaxies are virtually identical. This similarity has been noted by Schneider, Gunn, \& Hoessel (1983); they found that the $g-r$ colors of their sample of $\mathrm{cD}$ galaxies are highly invariant. To obtain a smooth continuum for Abell 1795, we scale the template spectra in amplitude and shift in velocity to match the object spectra, and then subtract it from the object continuum.

As Figure $2 a$ and $2 b$ show, the coronal line [Fe x] $6374 \AA$ we are searching for is located on the red wing of the strong nebular emission line [O I] $6363 \AA$. The ratio of transition probabilities for [O I] $6300 \AA$ and $\left[\begin{array}{ll}\mathrm{O}_{\mathrm{I}} & \mathrm{I}\end{array} 6363 \AA\right.$ is 3.0 (Osterbrock 1989). To search for the weak [Fe x] $6374 \AA$ emission line, we scale [O I] $6300 \AA$ down by a factor of 3 , shift it to the position of [O $\left.\mathrm{O}_{\mathrm{I}}\right] 6363 \AA$, and subtract if from the [O I] $6363 \AA$ line. Then we look for the $[\mathrm{Fe} \mathrm{X}] 6374 \AA$ emission line in the residual. Figure 4 shows this procedure; the bottom panel is the residual after the subtraction.

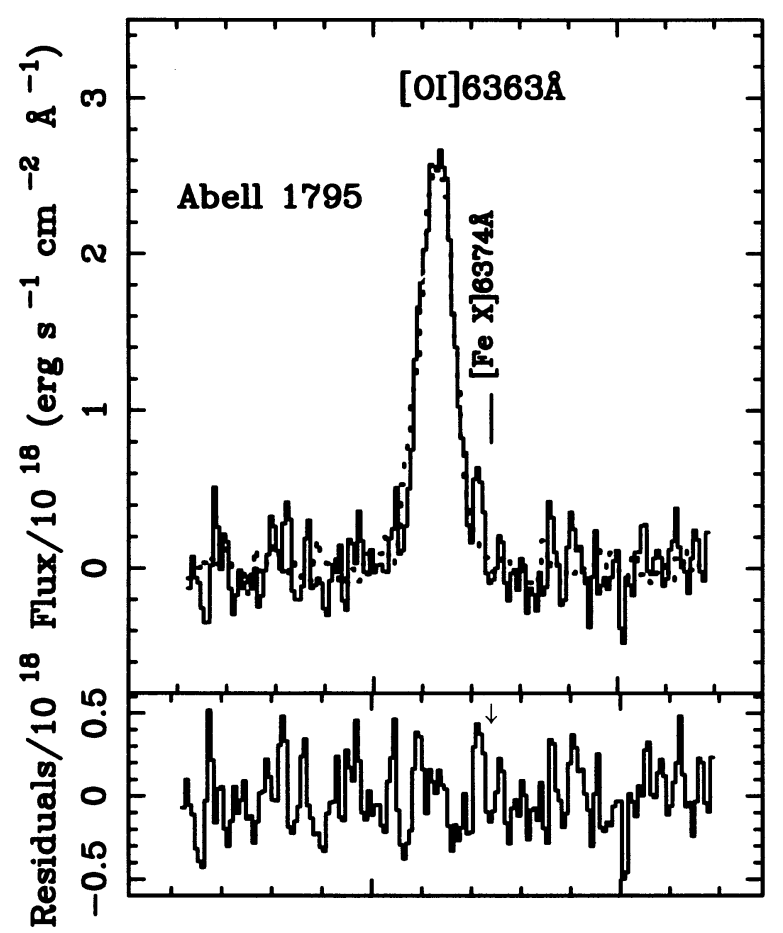

Observed Wavelength ( $($ )

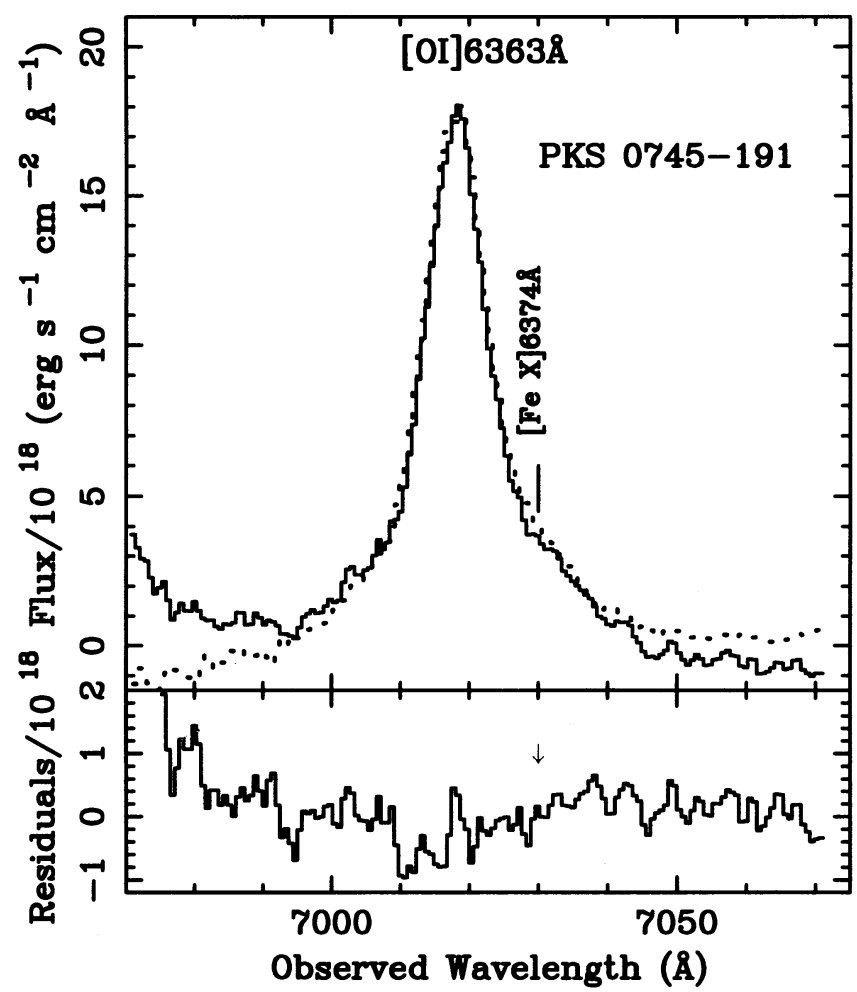

FIG. 4.-The top panels show the observed spectra in the region of the [Fe x] $6374 \AA$ line. The dotted lines represent the [O I] $6300 \AA$ line, which are scaled down by a factor of 3 (Osterbrock 1989) and shifted to the position of [O I $6363 \AA$. The [Fe x] $6374 \AA$ line is indicated right on the red wing of the strong emission line $\left[\mathrm{O}_{\mathrm{I}}\right] 6363 \AA$. The bottom panels show the residuals after the removal of [O I] $6363 \AA$. The arrows show the position where [Fe x] $6374 \AA$ is supposed to be. We did not detect any significant [ $\mathrm{Fe} \mathrm{x}]$ emission line in either Abell 1795 and PKS $0745-191$. 


\section{2. $P K S 0745-191$}

We apply basically the same method we used for analyzing Abell 1795 to PKS 0745-191. However, there is one difference between these two clusters. PKS 0745-191 does not show any rotation greater than $50 \mathrm{~km} \mathrm{~s}^{-1}$, as was also noted by Heckman et al. (1989). Furthermore, PKS 0745-191 is a strong active galactic nucleus (AGN) and the central active nucleus could also produce $[\mathrm{Fe} \mathrm{X}] 6374 \AA$ emission. Thus, we search for the $[\mathrm{Fe} \mathrm{X}] 6374 \AA$ in two regions: the central region with a radius of 2 ". 5 around the nucleus (e.g., $7 h_{50}^{-1} \mathrm{kpc}$ ) and the outer region between radii of 2 ". 5 to $5^{\prime \prime}$. This provides a means of distinguishing the possible [ $\mathrm{Fe} \mathrm{x}]$ coronal line produced by the central AGN activity from the coronal line originating in the cooling flow gas in the outer region. The outer radius of $5^{\prime \prime}$ is chosen to reach as far from the central nucleus as the signal in the spectrum permits us. We have four spectra of PKS 0745-191, with a total of $9500 \mathrm{~s}$ exposure. For both the central and outer regions, we examine each individual spectrum as well as the sum of all four spectra to search for the weak [Fe X] $6374 \AA$ line. The top panel of Figure 4 shows the spectrum region around [O I] $6363 \AA$ of the central nucleus after the continuum subtraction, and the bottom panel is the residual after the removal of the [O I $] 6363 \AA$ line.

\section{RESULTS}

We did not detect any significant $[\mathrm{Fe} \mathrm{x}] 6374 \AA$ line emission in the central several $10 \mathrm{kpc}$ regions of the two massive cooling flow clusters Abell 1795 and PKS 0745-191. The $1 \sigma$ surface brightness limits for the $[\mathrm{Fe} \mathrm{x}]$ emission line in the central $75 h_{50}^{-1} \mathrm{kpc}$ in Abel 1795 and in the central $30 h_{50}^{-1} \mathrm{kpc}$ region in PKS 0745-191 are $10^{-18}$ ergs s$^{-1} \mathrm{~cm}^{-2} \operatorname{arcsec}^{-2}$ and $7 \times 10^{-19}$ ergs $\mathrm{s}^{-1} \mathrm{~cm}^{-2} \operatorname{arcsec}^{-2}$, respectively. Our limits are much more sensitive than previous measurements in both clusters. They clearly rule out the homogeneous model with $\dot{M} \sim$ const in the central few $10 \mathrm{kpc}$ region of the cooling flow clusters. Our upper limit on the [Fe X] surface brightness is consistent with what is predicted by the popular inhomogeneous models (Sarazin \& Graney 1991), in which gas cools out of the flow at a range of radii, leaving the hot gas distributed as $\dot{M} \propto r$.

To derive the integrated mass cooling rate from the measured [Fe x] $6374 \AA$ surface brightness, we formulate $\dot{M}_{[\mathrm{Fe} \mathrm{X}]}$ as a function of observable quantities from equation (1):

$$
\dot{M}_{[\mathrm{Fe} \mathrm{X]}}(<\theta)=6 \times 10^{20}\left(\theta^{2} S\right) M_{\odot} \mathrm{yr}^{-1}
$$

Here $\theta$ is the radius in arcsecond of a circular region around the nucleus, $\dot{M}_{[\mathrm{Fe} \mathrm{X}]}(<\theta)$ is the integrated mass cooling rate within this region, and $S$ is the measured [Fe x] $6374 \AA$ surface brightness in ergs $\mathrm{s}^{-1} \mathrm{~cm}^{-2} \operatorname{arcsec}^{-2}$. We obtained the upper limit for the integrated mass cooling rate within a $10 \mathrm{kpc}$ radius of the Abell 1795 nucleus $\dot{M}_{[\mathrm{Fe} \mathrm{X]}}(<15 \mathrm{kpc})<170 h_{50}^{-2}$ $M_{\odot} \mathrm{yr}^{-1}$. Comparing with the integrated mass cooling rate within a $40 \mathrm{kpc}$ radius of the nucleus $\dot{M}_{X}(<40 \mathrm{kpc}) \sim 50 M_{\odot}$ $\mathrm{yr}^{-1}$ from $X$-ray observations (Edge et al. 1992), our result is consistent with X-ray observations. For PKS 0745-191, the derived $\dot{M}_{[\mathrm{Fe} \mathrm{X}]}(<15 \mathrm{kpc})<115 h_{50}^{-2} M_{\odot} \mathrm{yr}^{-1}$, and the mass cooling rate inferred from X-ray observations $\dot{M}_{X}(<240$ kpc) $\sim 400 M_{\odot} \mathrm{yr}^{-1}$. With lack of X-ray data on the central 10 $\mathrm{kpc}$ for PKS 0745-191, our result provides an upper limit on the integrated $\dot{M}$ within a $15 \mathrm{kpc}$ radius. It also suggests that less than $28 \%$ of the total mass cooling rate over an extent of $\sim 500 \mathrm{kpc}$ occurs in the central $30 \mathrm{kpc}$. Table 2 gives the properties derived from our observations.
In Abell 1795, we extracted a total 200 1D spectra, each with the aperture width of 1 pixel. Then we grouped and averaged these 1D spectra according to their distance and kinematics relative to the center, in the sense that the $1 \mathrm{D}$ spectra in the same group are spatially adjacent and have similar kinematic velocities relative to the center. We obtained 12 averaged spectra, and in each spectrum we searched for the weak [Fe $\mathrm{x}$ ] $6374 \AA$ line. This allows us to make an $1 \sigma$ map of the surface brightness limits for the [Fe X] $6374 \AA$ line in the central 42", i.e., $75 h_{50}^{-2} \mathrm{kpc}$ region in Abell 1795. The flux limit is calculated as follows: $F(1 \sigma)=\mathrm{rms} \times N^{1 / 2} \times d$, where rms is the standard deviation in the continuum of the residual spectrum after [O $\mathrm{I}]$ $6363 \AA$ is subtracted out, $N$ is the FWHM of the $[\mathrm{Fe} \mathrm{X}]$ line in pixels if the line were detected, and $d$ is the dispersion $(\AA$ per pixel). $\mathrm{Fe} x$ ions are much heavier than hydrogen. Thus its thermal velocity dispersion at temperature of $10^{6} \mathrm{~K}$ is much smaller than the random kinematic velocity of a few hundred $\mathrm{km} \mathrm{s}^{-1}$ in the central region of a cD galaxy. Since we do not know much about the kinematics of the coronal line emitting gas, we simply assume that the gas emitting [Fe x] $6374 \AA$ has similar kinematic motion as the optical nebulae and the FWHM of [Fe X] $6374 \AA$ is about the same as that of the [O I] $6363 \AA$ line. We measured the $1 \sigma$ upper limits of the $[\mathrm{Fe} \mathrm{X}]$ 6374 surface brightness profiles along both the major and minor axes. As Figure 5 shows, the solid dots are the limits along the minor axis, and the solid triangles along the major axis. In Figure 5, we also plot the theoretically predicted surface brightness of the $[\mathrm{Fe} \mathrm{x}]$ emission with the homoge-

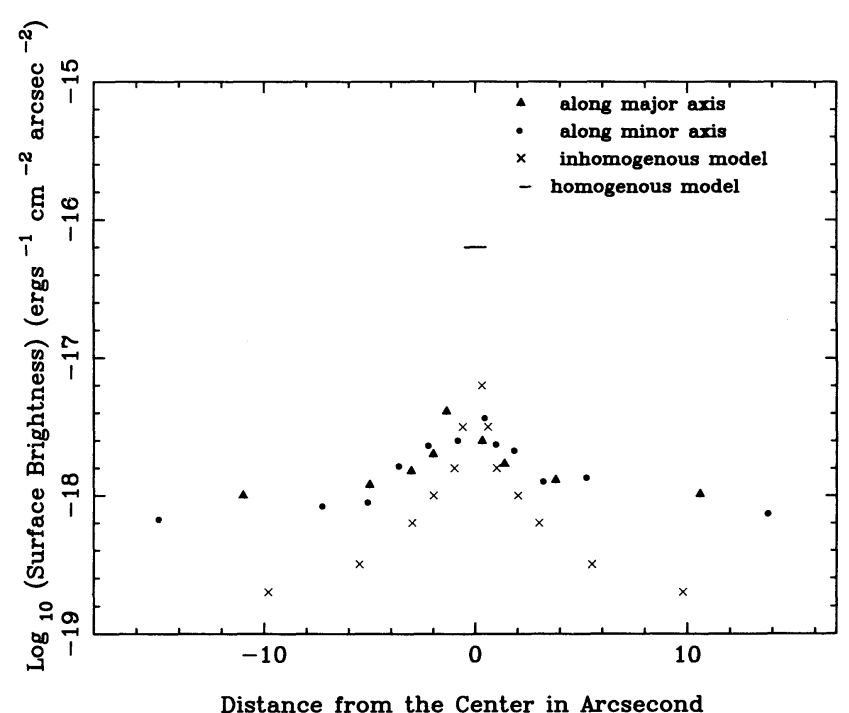

FIG. 5.-A map of $1 \sigma$ limit on the [Fe x] $6374 \AA$ line surface brightness in Abell 1795. The solid dots represent the measured [Fe x] $6374 \AA$ line surface brightness profile along the minor axis, and the triangles along major axis. Each of these solid and triangle points are measured by averaging a number of 1D spectra with aperture size of 1 pixel. See text for details. For comparison, we also plot the predicted surface brightness profiles with different theoretical models by Sarazin \& Graney (1991). For the homogeneous model with $\dot{M} \sim \sim_{17}$ const, the calculated surface brightness strongly peaks at the level of $6 \times 10^{-1}$ ergs $\mathrm{s}^{-1} \mathrm{~cm}^{-2}$ arcsec ${ }^{-2}$ with the central $1^{\prime \prime}$ region and drops dramatically to less than $10^{-19} \mathrm{ergs} \mathrm{s}^{-1} \mathrm{~cm}^{-2} \operatorname{arcsec}^{-2}$ beyond the central $1^{\prime \prime}$ region. In our plot, the short straight line indicates the predicted surface brightness with the homogeneous model within the central $1^{\prime \prime}$ region. The open squares show the predicted surface brightness profile with the inhomogeneous model with $\dot{M} \propto r$. Our result clearly rules out the $\dot{M} \sim$ const model in the central few 10 kpc of cooling flow clusters, for which X-ray observations of high spatial resolution do not yet exist. 
neous model of $\dot{M} \sim$ const and the inhomogeneous model of $\dot{M} \propto r$ (Sarazin \& Graney 1991). The open squares in the plot represent the predicted surface brightness profile with the inhomogeneous model with $\dot{M} \propto r$. For the homogeneous model with $\dot{M} \sim$ const, the calculated surface brightness strongly peaks at the level of $6 \times 10^{-17} \mathrm{ergs} \mathrm{s}^{-1} \mathrm{~cm}^{-2} \operatorname{arcsec}^{-2}$ within the central 1 " region and drops dramatically to less than $10^{-19}$ ergs $\mathrm{s}^{-1} \mathrm{~cm}^{-2} \operatorname{arcsec}^{-2}$ beyond the central $1^{\prime \prime}$ region. In our plot, the short straight line indicates the predicted surface brightness with the homogeneous model within the central $1^{\prime \prime}$ region. Also for comparison, Donahue \& Stocke's (1994) $1 \sigma$ surface brightness limit is $8 \times 10^{-18} \mathrm{ergs} \mathrm{s}^{-1} \mathrm{~cm}^{-2} \operatorname{arcsec}^{-2}$.

In addition, we also measured the upper limits on the [Fe $\mathrm{x}] / \mathrm{H} \alpha$ ratio within the central $30 \mathrm{kpc}$ region in both clusters Abell 1795 and PKS 0745-191. The $[\mathrm{Fe} \mathrm{x}] / \mathrm{H} \alpha(R<10 \mathrm{kpc})$ upper limit in Abell 1795 is $0.6 \%$ and $0.13 \%$ in PKS 0745-191. These upper limits are also tabulated in Table 2. The observed $[\mathrm{Fe} \mathrm{x}] / \mathrm{H} \alpha$ ratios put constraints on theoretical models, which take hot gas at an energy source for continuously reionizing the optical nebulae at temperature of $10^{4} \mathrm{~K}$ in order to explain the strong, low-ionization emission lines in cooling flow clusters.

\section{DISCUSSION}

The $[\mathrm{Fe} \mathrm{x}] 6374 \AA$ line luminosity directly measures the mass cooling rate of hot gas in the central regions of cooling flow clusters. Furthermore, the measured $[\mathrm{Fe} \mathrm{X}] / \mathrm{H} \alpha$ ratio provides a strong constraint on various theoretical models with cooling hot gas as an energy source for photoionizing the luminous, cool $\left(\sim 10^{4} \mathrm{~K}\right)$ nebular filaments.

We did not detect any significant $[\mathrm{Fe} \mathrm{x}] 6374 \AA$ coronal emission line in the central few $10 \mathrm{kpc}$ regions in massive cooling flow Abell 1795 and PKS 0745-191. Our upper limits on the surface brightness of the [Fe $\mathrm{X}]$ line are consistent with the X-ray model of inhomogeneous cooling flow with $\dot{M} \propto r$. In addition, our results contradict the previous positive detections in Abell 1795 reported by Anton et al. (1991) and in PKS 0745 - 191 by Donahue \& Stocke (1994). Our long-slit spectra of PKS 0745-191 were taken along the same slit position angle as that of Donahue \& Stocke's observations. Our measurements is an order of magnitude more sensitive and yielded no detection. In the case of Abell 1795, our observation may have been taken along the slit angles different from Anton et al.'s since their paper did not specify the slit position angles. This allows the same possibility that cooling gas in Abell 1795 may be very lumpy, and our slit just did not go through any cooling gas. However, Donahue \& Stocke (1994) also did not detect any [Fe $\mathrm{X}]$ emission in Abell 1795. (Our slit position angles are also different from those Donahue \& Stocke used.)
Our upper limits on the $[\mathrm{Fe} \mathrm{x}] / \mathrm{H} \alpha$ ratio put stringent constraints on theoretical models for production of the luminous low-ionization emission lines in cooling flow clusters by nebular heating. Any model which relies on hot gas to irradiate the nebulae has to satisfy the upper limits on $[\mathrm{Fe} \mathrm{x}] / \mathrm{H} \alpha$ we measured in these two clusters. Specifically, the Voit \& Donahue (1990) photoionization model for high column density clouds requires an emission-line ratio of $[\mathrm{Fe} \mathrm{x}] / \mathrm{H} \alpha \sim$ $0.0032 f_{c}^{-1}$, where $f_{c}$ is the covering factor. With our $[\mathrm{Fe} \mathrm{x}] / \mathrm{H} \alpha$ limits, the covering factors for Abell 1795 and PKS $0745-191$ are $\geq 0.5$ and $\geq 2.4$, respectively. Since the covering factor by definition is $\leq 1$, this suggests that our measurement of the [Fe $\mathrm{x}] / \mathrm{H} \alpha$ ratio in PKS $0745-191$ rules out the Voit \& Donahue photoionization model.

Our results impose the same constraint on other photoionization models such as turbulent mixing layers (Begelman \& Fabian 1990; Crawford \& Fabian 1992), thermal conduction model (Sparks, Macchetto, \& Golombek 1989; de Jong et al. 1990), and fast shocks (Binette, Dopita, \& Tuohy 1985), etc. In all these models the thermal energy of the hot intracluster medium is tapped by creating gas at intermediate temperatures $\left(10^{5.5}-10^{6.5} \mathrm{~K}\right)$, from which ultraviolet and soft $\mathrm{X}$-ray radiation provides the photons ionizing the cool clouds $\left(10^{4} \mathrm{~K}\right)$. Thus $[\mathrm{Fe} \mathrm{X}] 6374 \AA$ line surface brightness and $[\mathrm{Fe} \mathrm{X}] / \mathrm{H} \alpha$ ratio can be predicted with all these models. However, none of these models except the Voit \& Donahue photoionization model (Voit et al. 1994) has given detailed calculations of expected $[\mathrm{Fe} \mathrm{x}] 6374 \AA$ surface brightness and $[\mathrm{Fe} \mathrm{x}] / \mathrm{H} \alpha$ ratio. It would be very interesting to do the calculations with these models to compare with our measurements. For instance, in the fast shocks model (Binette et al. 1985) gas is heated up to nearly X-ray emitting temperatures, which then cools and irradiates the cool gas $\left(10^{4} \mathrm{~K}\right)$. The upper limits on $[\mathrm{Fe} \mathrm{x}] / \mathrm{H} \alpha$ ratio from our measurements set the maximum shock velocity allowed in this model. The calculations done by $\mathbf{M}$. Donahue (private communication) have shown that our measurements convincingly rule out high-velocity shocks which can heat the gas to temperatures greater than $10^{6} \mathrm{~K}$, i.e., shocks with velocities greater than $400 \mathrm{~km} \mathrm{~s}^{-1}$ in the Binette et al. (1985) model.

The W. M. Keck Observatory is a scientific partnership between the California Institute of Technology and the University of California. It was made possible by the generous and visionary gift by the W. M. Keck foundation, and the support of its president, Howard Keck. It is a pleasure to thank the observing assistants T. Chelminiak and B. Schaefer for their expert work during the observing runs. We wish to thank Megan Donahue for the useful discussions and her constructive suggestions, which have helped us to improve the paper.

\section{REFERENCES}

Anton, K., Wagner, S., \& Appenzeller, I. 1991, A\&A, L51

Baum, S. A. 1992, in Clusters and Superclusters of Galaxies, ed. A. C. Fabian (Dordrecht: Kluwer), 171

Begelman, M. C., \& Fabian, A. C. 1990, MNRAS, 244, 26

Binette, L., Dopita, M. A., \& Tuohy, I. R. 1985, ApJ, 297, 476

Canizares, C. R., Markert, T. M., \& Donahue, M. 1988, in Cooling Flows in Clusters and Galaxies, ed. A. C. Fabian (Dordrecht: Kluwer), 61

Cowie, L. L. 1981, in X-Ray Astronomy with the Einstein Satellite, ed. R. Giacconi (Dordrecht: Kluwer), 227

de Jong, T., Norgaard-Nielson, H. U., Jorgensen, H. E., \& Hansen, L. 1990, A\&A, 232, 317

Donahue, M., \& Stocke, J. T. 1994, ApJ, 422, 459

Edge, A. C., \& Stewart, G. C. 1991, MNRAS 252, 414

Edge, A. C., Stewart, G. C., \& Fabian, A. C. 1992, MNRAS, 258, 177

Heckman, T., Baum, S. A., van Breugel, W. J. M., \& McCarthy, P. 1989, ApK, 338,48
Hu, E. M., Cowie, L. L., \& Wang, Z., 1985, ApJS, 59, 447

Mushotzky, R. 1992, in Clusters and Superclusters of Galaxies, ed. A. C Fabian (Dordrecht: Kluwer), 91

Mushotzky, R., \& Szymkowiak, M. 1988, in Cooling Flows in Clusters and Galaxies, ed. A. C. Fabian (Dordrecht: Kluwer), 53

Oke, J. 1990, AJ, 99, 1621

Oke, J. B., et al. 1995, PASP, in press

Osterbrock, D. E. 1989, Astrophysics of Gaseous Nebulae and Active Galactic Nuclei (Mill Valley: University Science Books)

Sarazin, C. L., \& Graney, C. M. 1991, ApJ, 375, 532

Shields, J. C., \& Filippenko, A. V. 1992, AJ, 103, 1443

Schneider, D. P., Gunn, J. E., \& Hoessel, J. G. 1983, ApJ, 268, 476

Sparks, W. B., Macchetto, F., \& Golombek, D. 1989, ApJ, 345, 153

Tonry, J., \& Davis, M. 1979, AJ, 84, 1511

Voit, G. M., \& Donahue, M. 1990, ApJ, 390, L15

Voit, G. M., Donahue, M., \& Slavin, J. D. 1994, ApJS, 95, 87 